\title{
Edentulism and associated variables among institutionalized elders
}

\begin{abstract}
Objectives: To evaluate the association between edentulism and sociodemographic characteristics, cognitive function, independence in activities of daily living, presence of chronic diseases, medications and oral mucosa changes among 160 institutionalized elders (mean age of 77.1 \pm 11.1 years) from a city of Brazil.
\end{abstract}

Methods: Data were collected by a calibrated dentist in a clinical examination (DMFT index and oral mucosa changes) and by a trained researcher in an interview. Statistical analysis was performed by association tests and multiple logistic regression $(\mathrm{p}<0.05)$.

Results: The mean DMFT was $25.9(\mathrm{SD}=4.2)$. About $60.6 \%$ were edentulous and $87.5 \%$ had cognitive impairment. Coated tongue and candidiasis were the most prevalent mucosa changes. Female $(\mathrm{OR}=2.1)$ or totally dependent $(\mathrm{OR}=2.5)$ or using up to two drugs $(\mathrm{OR}=2.7)$ individuals were more likely to have edentulism $(\mathrm{p}<0.05)$.

Conclusion: It was concluded that the predictors of edentulism for the studied sample were female sex, use of up to two medications per day and total dependence on activities of daily living.

Keywords: ageing, institutionalized elderly, oral health, long-term care, tooth loss

Volume 3 Issue 5 - 2018

Andréia Affonso Barretto Montandon, Lígia
Antunes Pereira Pinelli,Vanessa Pardi, Elaine
Pereira da Silva Tagliaferro, Laiza Maria Grassi
Fais
Faculty of Dentistry, University Araraquara Dental School, Brazil

Faculty of Dentistry, University Araraquara Dental School, Bra

Correspondence: Andréia Affonso Barretto Montandon, Designation Assistante Professor, University Araraquara Dental School, Univ Estadual Paulista, Brazil, Tel +55I6330I 6343, Email andreiam@foar.unesp.br

Received: August 0, 2018| Published: September 10, 2018

\section{Introduction}

The population aging is a worldwide phenomenon. It is estimated that the number of people aged 60 or more will reach two billion people or $22 \%$ of the global population by $2050,{ }^{1}$ which may indicate that the number of institutionalized elders will also increase. Usually, institutionalized patients have high degree of dependency that can contribute to the poor oral health and unfavorable local physiological conditions. $^{2}$ In this respect, attention to these unfavorable oral conditions should be priority for the institution staff. ${ }^{3}$

The retention or loss of permanent teeth is of central importance to an individual's oral health status and to quality of life. ${ }^{4}$ Several studies have evidenced the poor oral health of institutionalized elderly people such as the common presence of edentulism. ${ }^{5,6}$

The severity of dental caries in this population has been represented by high DMFT values, ranging from 23 to $30.8,{ }^{2}$ by the presence of biofilm in most tooth surfaces ${ }^{2}$ as well as by the largest need for restorative and periodontal care. ${ }^{5}$ Frenkel, Harvey, \& Newcombe ${ }^{7}$ (2000) found that $33 \%$ of the elders in United Kington had stomatitis and most of them ( $82 \%$ ) were proved unable to effectively clean their dentures.

Moreover, in long-stay institutions it is very difficult to provide adequate oral health for the elder due to multiple factors, such as the low number of caregivers and their low educational level, lack of dental assistance, inappropriate hygiene ${ }^{8}$ the reduced dexterity, ${ }^{9}$ and the cost of dental care in an already overburdened health sector. ${ }^{10}$ Some authors draw attention to the institutionalization as a risk factor for the inappropriate or suboptimal drug utilization of drugs, particularly of psychotropic drugs. ${ }^{11,12}$

Another important aspect is the influence of cognitive impairment on oral health. The presence of tooth loss, gingival bleeding, periodontal attachment loss, ${ }^{13}$ dental caries and absence of dentures ${ }^{14}$ has been associated with worse cognitive function. On the other hand, low risk of periodontal disease was associated with higher scores of cognitive functions among non-institutionalized elders, ${ }^{15}$ In addition, general health problems are frequent, variable, coexistent, necessitating the daily administration of multiple medications, while oral examination is an essential part of a multidisciplinary approach in elders. ${ }^{16}$

The aim of this cross-sectional study was to evaluate the association between edentulism and sociodemographic, cognitive, independence in activities of daily living, presence of chronic diseases, medications and oral mucosa changes among elders from long-term care institution (LTC) in a city in the southeastern region of Brazil.

\section{Methods}

This study was conducted after being approved by the Ethics Committee of the Araraquara Dental School - UNESP (Protocol No. 46/07) and was conducted in full accordance with the World Medical Association Declaration of Helsinki. An informed consent form was signed by participants or, in case of total disability, by their family members or by the institution's manager, before starting data collection. Patient anonymity was preserved.

This was an observational cross-sectional study conducted in a long-term care institution were the elders were examined by a dentist. Data about socio-demographics, cognitive status, independence in activities of daily living, presence of chronic diseases, habits and knowledge of oral health and medications were obtained by means of an interview.

In this cross-sectional study, all people aged 60 years or more $(n=187)$ from the largest long-term institution from Araraquara, a city in the southeastern region of Brazil, were invited to participate. The inclusion criteria were the signed informed consent and aged 60 years or more. The exclusion criterion was the refusal to participate in the 
study. Among all institutionalized elders, 27 refused to participate, totaling 160 individuals.

The clinical examinations and the interviews with the elders and caregivers were performed in a period of eight months. The interviewer was trained for the study through classroom instruction and self-study to performing interviews in a professional manner, certifying the quality of collected data, and avoiding influencing the patients' responses. Health records were also assessed for additional information. Questions included information on socio-demographics, cognitive status, independence in activities of daily living, presence of chronic diseases, habits and knowledge of oral health and medications taken, as detailed below:

a. Socio-demographic variables: age, marital status, educational level, type of institutionalization (private or public) and length of stay.

b. Cognitive assessment: assessed with the Mini Mental State Examination-MMSE. ${ }^{17}$ It includes dimensions of orientation, attention, memory, language and visual-spatial skills. The MMSE has maximum score of 30 with a point for each question. A standard cut-point of $\leq 23$ was considered for cognitive impairment. ${ }^{18}$ Additionally, a second MMSE score was determined considering the educational level of each patient, since the literature has shown a strong relationship between education and MMSE scores. ${ }^{18}$

Functional capacity evaluation (Activities of Daily Living ADLs): Functional status was assessed by the Katz ADL Index. ${ }^{19}$ Basic Activities of Daily Living (BADL) which measures basic vital functions that are the last one to be lost during the aging process and corresponds to seven items related to personal care, feeding, continence, bathing, dressing, transferring from the bed to the chair and walk across the room. The Instrumental Activities of Daily Living (IADL) Scale ${ }^{20}$ was used to determine the more complex and initially lost activities corresponding to eight items: using the telephone, using transport outside the home, shopping market, preparing meals, performing housework, washing, handling of drugs and finance. For each activity, it was assigned one point when there was no dependence, two points if he/she needed any help and, three points if there was complete dependence. For BADL, scores 7 and 21 indicated complete independence and complete dependence, respectively; for IADL, complete independence had sum equal to 8 and complete dependence, sum equal to 24 . The participants were classified in independent, partially dependent and totally dependent.

The oral health status was assessed according to the WHO criteria. ${ }^{21}$ A single trained examiner collected data on tooth loss, dental caries (DMFT index), root caries, use and needs of prosthesis and lesions of oral mucosa and soft tissues in and around the mouth.

The prosthetic status was recorded for both dental jaws, considering the following criteria: no prosthesis, fixed partial denture, more than one fixed partial denture, removable partial denture, both fixed and removable partial denture and complete denture. ${ }^{21}$ The prosthetic need was also recorded for both dental jaws, according to the following criteria: no prosthetic needed, need for crowns (one tooth replacement), need for fixed partial dentures, need for a combination of crows and/or fixed partial dentures, need for complete. ${ }^{21}$ It is important to mention that those patients who needed new prosthesis were rehabilitated.

Changes in the oral mucosa and soft tissues in and around the mouth were recorded as follows: no abnormal condition; malignant tumor (oral cancer); leukoplakia; lichen planus; ulceration (aphthous, herpetic, traumatic); acute necrotizing ulcerative gingivitis; candidiasis; abscesses; other conditions. ${ }^{21}$

During data analysis, edentulism (total tooth loss) was considered the dependent variable. Bivariate analyses by Chi-square or Fisher's exact tests were performed to assess the association of interest. Those with $\mathrm{p} \leq 0.20$ were selected to be included in multivariate logistic regression with stepwise procedure. The odds ratio (OR) and corresponding $95 \%$ confidence intervals (CI) were estimated for the variables remained in the multiple regression model. All statistical tests were performed using SAS program, adopting a significance level of $5 \%$.

\section{Results}

In this study participants were aged $60-102$ years (mean age of $77.1 \pm 11.1$ years $)$. Most were women $(n=104 ; 65.0 \%)$ and considered of low socioeconomic level $(n=122 ; 76.3 \%)$ by the long-stay institution. Table 1 shows socio-demographic characteristics of the participants.

Table I Sociodemographics characteristics of the studied population $(n=160)$

\begin{tabular}{lll}
\hline Variable & Category & n (\%) \\
\hline Age group (years) & $60-64$ & $28(17.5)$ \\
& $65-74$ & $35(21.9)$ \\
& $75-84$ & $49(30.6)$ \\
Marital status & $85--+$ & $48(30.0)$ \\
& Married & $22(13.7)$ \\
& Single & $70(43.8)$ \\
& Divorced & $16(10.0)$ \\
& Widower & $52(32.5)$ \\
& Illiterate & $66(41.3)$ \\
& Incomplete elementary school & $72(45.0)$ \\
& Complete elementary school & $09(5.6)$ \\
& Incomplete high school & $02(1.3)$ \\
& Complete high school & $07(4.4)$ \\
& Incomplete undergraduate studies & $01(0.6)$ \\
& Complete undergraduate studies & $03(1.8)$ \\
\hline
\end{tabular}

Considering the presence of chronic diseases, almost all the participants $(\mathrm{n}=156 ; 97.5 \%)$ had at least one disease. According to the health team staff or the medical records, 34 subjects $(21.3 \%)$ were in a regular medical treatment for Hypertension (HBP). Most participants $(75.5 \%)$ used drugs on a regular basis, and $60.5 \%$ used one to four different ones daily. On average, each participant ingested 2.3 different types of drugs a day.

In relation to oral health status, about two-thirds were edentulous $(\mathrm{n}=97 ; 60.6 \%)$ and the mean number of teeth was 4.2. When considering only those with at least one tooth $(n=63)$, the mean number of teeth present was 10.8. The mean (SD) DMFT index was 25.9 (4.2). The maximum score (32) of the DMFT was found among $66.4 \%$ participants and only seven people $(4.4 \%)$ had DMFT score $\leq 15$. On average, root caries was detected in 2.4 teeth among those with at least one tooth. Considering those with at least one tooth $(n=63), 80.9 \%$ required basic periodontal treatment, $31.7 \%$ extractions and $38 \%$ restorative procedures. Regarding changes in the oral mucosa and soft tissues in and around the mouth, $47.3 \%$ presented coated tongue, $45.0 \%$ candidiasis and $7.7 \%$ angular cheilitis. 
Cognitive impairment detected by the Mini Mental State Examination (MMSE), was present in $71.9 \%$ of patients $(n=115)$, when the level of schooling was considered. ${ }^{18}$ However, when the original methodology ${ }^{17}$ was used for patients' classification, $87.5 \%$ of the participants had cognitive impairment.

Table 2 shows the elders distribution according to cognitive impairment and MMSE mean score. Table 3 shows the distribution of participants in relation to the degree of functional impairment in Basic Activities of Daily Living and Instrumental Activities of Daily Living.

Table 2 Distribution of institutionalized elders with cognitive impairment, according to mean score of Mini Mental State Examination (MMSE) and age group.

\begin{tabular}{lll}
\hline Age group & $\mathbf{n}(\%)$ & mean MMSE \\
\hline $60-64$ & $14(12.1)$ & 14.6 \\
$65-74$ & $22(19.1)$ & 11.4 \\
$75-84$ & $36(31.4)$ & 10.7 \\
$85-+$ & $43(37.4)$ & 4.8 \\
Total & $115(100.0)$ & 10.3 \\
\hline
\end{tabular}

Table 3 Distribution (\%) of institutionalized elders according to Basic Activities of Daily Living (BADL) and Instrumental Activities of Daily Living (IADL).

\begin{tabular}{lll}
\hline Variable & BADL & IADL \\
\hline Independent & 30.6 & 9.4 \\
Partially dependent & 44.3 & 29.4 \\
Totally dependent & 25.1 & 61.2 \\
Total & 100.0 & 100.0
\end{tabular}

Table 4 and Table 5 show the results of bivariate and logistic regression analyses, respectively. Females participants $(\mathrm{OR}=2.1)$ had more likely to be edentulous than male. Those who used up to two drugs $(\mathrm{OR}=2.7)$ were more likely to be edentulous than those who used three or more drugs. The probability of those totally dependents being edentulous was 2.5 times greater than that of partially dependent or independently.

Table 4 Associaton between edentulism and variables of interest

\begin{tabular}{|c|c|c|c|c|}
\hline \multirow{3}{*}{ Variables } & \multicolumn{2}{|l|}{ Edentulism } & \multirow{3}{*}{ Unadjusted OR (Cl 95\%) } & \multirow{3}{*}{$\mathbf{p}$} \\
\hline & Yes & No & & \\
\hline & $\mathrm{n}(\%)$ & $\mathrm{n}(\%)$ & & \\
\hline \multicolumn{5}{|l|}{ Cognitive Impairment } \\
\hline$\leq 23$ & $90(63.38)$ & $52(36.62)$ & $2.72(0.99-7.44)$ & 0.0451 \\
\hline$>23$ & 7 (38.89) & II (6I.II) & $\mathrm{I}$ & \\
\hline \multicolumn{5}{|l|}{ Cognitive Impairment - Schooling } \\
\hline No & $22(48.89)$ & $23(5 I .11)$ & $0.5 I(0.25-1.03)$ & 0.0573 \\
\hline Yes & $75(65.22)$ & $40(34.78)$ & I & \\
\hline \multicolumn{5}{|l|}{ Dependence } \\
\hline Independent and partially dependent & $31(50.00)$ & $31(50.00)$ & $0.48(0.25-0.93)$ & 0.0287 \\
\hline Totally dependent & $66(67.35)$ & $32(32.65)$ & 1 & \\
\hline \multicolumn{5}{|l|}{ Illiteracy } \\
\hline Yes & $45(68.18)$ & $21(31.82)$ & I.73 (0.89-3.34) & 0.1011 \\
\hline No & $52(55.32)$ & $42(44.68)$ & $\mathrm{I}$ & \\
\hline \multicolumn{5}{|l|}{ Age } \\
\hline$\leq 78$ years & $46(52.87)$ & $41(47.13)$ & $0.48(0.25-0.93)$ & 0.0285 \\
\hline$>78$ years & $51(69.86)$ & $22(30.14)$ & I & \\
\hline \multicolumn{5}{|l|}{ Gender } \\
\hline Male & $27(48.21)$ & $29(51.79)$ & $0.45(0.23-0.88)$ & 0.0184 \\
\hline Female & $70(67.31)$ & 34 (32.69) & I & \\
\hline \multicolumn{5}{|l|}{ Low socioeconomic level } \\
\hline Yes & $75(61.48)$ & $47(38.52)$ & I.I $6(0.55-2.43)$ & 0.6932 \\
\hline No & $22(57.89)$ & $16(42.11)$ & $\mathrm{I}$ & \\
\hline \multicolumn{5}{|l|}{ Diseases } \\
\hline No & $19(61.29)$ & $12(38.71)$ & $\mathrm{I} .04(0.46-2.3 \mathrm{I})$ & 0.9327 \\
\hline
\end{tabular}


Table continued.

\begin{tabular}{|c|c|c|c|c|}
\hline Variables & Edentulism & & Unadjusted OR (Cl 95\%) & $\mathbf{p}$ \\
\hline Yes & $78(60.47)$ & $51(39.53)$ & 1 & \\
\hline \multicolumn{5}{|l|}{ Drugs use } \\
\hline Up to 2 drugs & $63(67.02)$ & 31 (32.98) & I.9I (I.00-3.65) & 0.0481 \\
\hline More than 2 drugs & $34(5 \mid .52)$ & $32(48.48)$ & I & \\
\hline \multicolumn{5}{|l|}{ Mental disorder } \\
\hline No & 61 (65.59) & $32(34.4 I)$ & $1.64(0.86-3.12)$ & 0.1298 \\
\hline Yes & $36(53.73)$ & 31 (46.27) & 1 & \\
\hline \multicolumn{5}{|l|}{ Cardiovascular disease } \\
\hline No & $58(59.18)$ & $40(40.82)$ & $0.86(0.44-1.64)$ & 0.639 \\
\hline Yes & $39(62.90)$ & $23(37.10)$ & 1 & \\
\hline \multicolumn{5}{|c|}{ Regular cardiovascular treatment } \\
\hline No & $77(6 \mathrm{I} . \mathrm{II})$ & 49 (38.89) & I. $10(0.5 \mathrm{I}-2.38)$ & 0.8086 \\
\hline Yes & $20(58.82)$ & $14(4|| 8)$. & $\mathrm{I}$ & \\
\hline \multicolumn{5}{|l|}{ Coated tongue } \\
\hline No & 73 (62.39) & $44(37.6 I)$ & I.3I (0.65-2.67) & 0.4502 \\
\hline Yes & $24(55.8 I)$ & $19(44.19)$ & I & \\
\hline \multicolumn{5}{|l|}{ Angular cheilitis } \\
\hline No & $94(6 \mathrm{I} .44)$ & $59(38.56)$ & $2.12(0.46-9.83)$ & 0.435 \\
\hline Yes & $3(42.86)$ & $4(57.14)$ & 1 & \\
\hline \multicolumn{5}{|l|}{ Candidiasis } \\
\hline No & $79(57.66)$ & $58(42.34)$ & $0.38(0.13-1.08)$ & 0.0614 \\
\hline Yes & $18(78.26)$ & $5(21.74)$ & I & \\
\hline
\end{tabular}

Table $\mathbf{5}$ Logistic regression with edentulism as dependent variable

\begin{tabular}{|c|c|c|c|c|}
\hline Variable & Edentulism n (\%) & Odds ratio & Confidence Interval 95\% & $\mathbf{p}$ \\
\hline \multicolumn{5}{|l|}{ Gender } \\
\hline Male & $27(48.2 I)$ & 1 & & 0.0184 \\
\hline Female & $70(67.31)$ & 2.1 & $1.04-4.24$ & \\
\hline \multicolumn{5}{|l|}{ Drugs use } \\
\hline Up 2 drugs & $63(67.02)$ & 2.7 & $1.33-5.66$ & 0.0315 \\
\hline More than 2 drugs & $34(5 \mathrm{I} .52)$ & 1 & & \\
\hline \multicolumn{5}{|l|}{ Dependence } \\
\hline Independent and partially dependent & $31(50.00)$ & 1 & & 0.013 \\
\hline Totally dependent & $66(67.35)$ & 2.5 & $|.22-5.2|$ & \\
\hline
\end{tabular}

\section{Discussion}

This study was designed to approach the association between edentulism and some associated variables in Brazilian institutionalized elders due to the limited knowledge about it. One of the variables studied is the cognitive impairment, in this aspect, the results of this study indicated that the higher the age, the higher the prevalence and severity of cognitive impairment. However, the average MMSE score for the study population was 10.3 , which can be considered low.

Almost all of participants $(90.6 \%)$ showed some dependence on the more complex activities and $69.4 \%$ presented impairment in activities for the survival, such as eating, grooming and locomotion, which indicates the high demand for services in the Institution. Therefore, the participants of this study were considered to have a high degree of fragility and dependence. These states may have complex psychosocial changes and contribute to greater vulnerability to oral pathologies. ${ }^{22}$

It has been suggested that the low number of caregivers and their low educational level ${ }^{8}$ may hamper the appropriate oral health care in long-stay institutions. Additionally, the common problems among dependent elderly population, such as their dependence in self-care oral hygiene, ${ }^{9,14}$ contributes to the poor oral health usually found in institutionalized elders..$^{2,5,22,23}$ 
In relation to oral health, our results demonstrated that most participants $(60.6 \%)$ were edentulous and that DMFT (25.9) was very high, indicating the poor oral health of the examined elders, as previously reported. ${ }^{2,3,5}$ Among the participants with at least one tooth $(n=63)$, the need for basic periodontal treatment was observed in $80.9 \%$ of them. They also needed extraction $(31.7 \%)$ and restorative care $(38 \%)$, which were similar to other findings. ${ }^{5}$ The most frequent lesion found in the participants was coated tongue $(47.3 \%)$, which is a common finding among institutionalized elders. ${ }^{23}$ These specific needs emphasize the importance of dental care planning target to institutionalized elderly people.

Among all the studied variables, gender, drugs use and dependence in daily activities remained in the final logistic regression model at $\mathrm{p}<0.05$. Females had more chance of edentulism, showing women as an important risk indicator of edentulism. ${ }^{6}$ It has been suggested that in many populations women had and continue to have higher rates of tooth loss and edentulism ${ }^{4}$. Moreover, women may have less wealth and lower income, which are closely related to the ability to pay for dental care and the type of dental care. ${ }^{4}$ Therefore, actions targeting the improvement of oral health and access to dental care should consider this important epidemiologic aspect.

This study also demonstrated that totally dependent elders were more prone to have edentulism. As pointed out by Gil-Montoya et al., ${ }^{3}$ health policies for older people with severe functional dependence should direct special attention to oral health, especially because, according to the authors, the practices of oral hygiene are not a priority for caregivers. Considering the need for comprehensive health care to the elder and the systemic benefits of good oral health status ${ }^{16}$ it is crucial that the institutions have a health team qualified for the basic oral hygiene care of the elder. By training caregivers who value oral health as an integral part of health promotion programs for the elders could be an important strategy to be implemented.

Finally, the logistic regression also showed that elderly people who ingested up to two drugs daily were more likely to have edentulism in comparison with those who take three or more drugs. There are three possible explanations for this occurrence: first, this result may be typical of the studied population, i.e., institutionalized elders; second, less drugs intake may not mean less need for medication use and finally, the number of drugs that are reported not always reflect the exact protocol of medication prescriptions since the literature have shown lack of treatment protocols in LTC institutions for the elder. ${ }^{11,12}$ It has been suggested that dental treatment of subjects with chronic diseases and multiple drugs use is, in general, more complex ${ }^{10}$ and the oral health team should be aware of this peculiarity.

There were some limitations in this study such as the crosssectional design of the study that does not allow establishing causality, since exposure and disease are assessed at the same time and the lack of external validity of the data, once the results refer to a specific population.

Despite these limitations, the authors believe that the findings of this study may contribute to the health staff and caregivers of the long-stay institution being able to prioritize the oral health care of the elderly women with total dependence since their admission.

\section{Conclusion}

It was concluded that the predictors of edentulism for the studied sample were female sex, use of up to two medications per day and total dependence on activities of daily living.

\section{Acknowledgements}

The authors thank FAPESP for financial support (Grant \# 2008111592), the LTC institution and the elders who participated in this study.

\section{Conflict of interest}

The author declares no conflict of interest.

\section{References}

1. Ageing in the twenty-first century: a celebration and a challenge. United Nations Population Fund. 2012.

2. Ferreira RC, Magalhães CS, Rocha ES, et al. Oral health among institutionalized elderly in Belo Horizonte, Minas Gerais, Brazil. Cad Saude Publica. 2009;25(11):2375-2385.

3. Gil MJA, De Mello ALF, Cardenas CB, et al. Oral health protocol for the dependent institutionalized elderly. Geriatr Nurs. 2006;27(2):95-101.

4. Russell SL, Gordon S, Lukacs JR, et al. Sex/Gender differences in tooth loss and edentulism: historical perspectives, biological factors, and sociologic reasons. Dent Clin North Am. 2013;57(2):317-337.

5. Carter G, Lee M, McKelvev V, Sourial A, et al. Oral health status and oral treatment needs of dependent elderly people in Christchurch. $N Z$ Med J. 2004;117(1194):U892.

6. Gaio EJ, Hass AN, Carrard VC, et al. Oral health status elders from South Brazil: a population based study. Gerodontology. 2012;29(3):214-223.

7. Frenkel H, Harvey I, Newcombe RG. Oral health care among nursing home residents in Avon. Gerodontology. 2000;17(1):33-38.

8. Vanobbergen JN, De Visschere LM. Factors contributing to the variation in oral hygiene practices and facilities in long-term care institutions for the elderly. Community Dent Health. 2005;22(4):260-265.

9. Padilha DM, Hugo FN, Hilgert JB, et al. Hand function and oral hygiene in older institutionalized Brazilians. J Am Geriatr Soc. 2007;55(9):13331338 .

10. Fejerskov O, Escobar G, Jossing M, et al. A functional natural dentition for all - and for life? The oral healthcare system needs revision. J Oral Rehabil. 2013;40(9):707-722.

11. Morin L, Fastbom J, Laroche ML, et al. Potentially inappropriate drug use in older people: a nationwide comparison of different explicit criteria for population-based estimates. Br J Clin Pharmacol. 2015;80(2):315-324.

12. Stroka MA. Drug overprescription in nursing homes: an empirical evaluation of administrative data. Eur J Health Econ. 2015. 17(3):257267.

13. Stewart R, Sabbah W, Tsakos GD, et al. Oral health and cognitive function in the third national health and nutrition examination survey (NHANES III). Psychosom Med. 2008;70(8):936-941.

14. Syrjälä AM, Ylostalo P, Sulkava R, et al. Relationship between cognitive impairment and oral health: results of the Health 2000 Health Examination Survey in Finland. Acta Odontol Scand. 2007;65:103-108.

15. Yu YH, Kuo HK. Association between cognitive function and periodontal disease in older adults. J Am Geriatri Soc. 2008;56(9):1693-1697.

16. Triantos D. Intra-oral findings and general health conditions among institutionalized and non-institutionalized elderly in Greece. J Oral Pathol Med. 2005;34(10):577-582.

17. Folstein MF, Folstein SE, McHugh PR. "Mini-mental state": a practical method for grading the cognitive state of patients for the clinican. $J$ Psiquiatr Res. 1975;12(3):189-198. 
18. Bertollucci PHF, Brucki SMD, Campacci S, et al. The Mini-Mental State Examination in an outpatient population: influence of literacy. Arq Neuropsiquiatr.1994;52(1):1-7.

19. Katz S, Dows TD, Cash HR, et al. Progress in development of the index of ADL. Gerontologist. 1970;10(1):20-30.

20. Lawton MP, Brody EM. Assessment of older people: self-maintaining and instrumental activities of dialing living. Gerontologist 1969;9(3):179-186.
21. Oral health surveys-basic methods. World Health Organization. $4^{\text {th }}$ ed. Geneva: WHO. 1997.

22. MacEntee MI, Nolan A, Thomason JM. Oral mucosal and osseous disorders in frail elders. Gerodontology. 2004;21(2):78-84.

23. Niessen LC, Fedele DJ. Aging successfully: oral health for the prime of life. Compend Contin Educ Dent. 2002;23(10 Suppl):4-11. 